\title{
What Is a Good Plan? Cultural Variations in Expert Planners' Concepts of Plan Quality
}

\author{
Louise J. Rasmussen \\ Winston R. Sieck \\ Applied Research Associates
}

\section{Paul Smart}

University of Southampton

АвSTRACT: This article presents the results of a field research study examining commonalities and differences between American and British operational planners' mental models of planning. We conducted cultural network analysis interviews with 14 experienced operational planners in the United States and the United Kingdom. Our results demonstrate the existence of fundamental differences between the ways American and British expert planners conceive of a high-quality plan. Our results revealed that the American planners' model focused on specification of action to achieve synchronization, providing little autonomy at the level of execution, and included the belief that increasing contingencies reduces risk. The British planners' model stressed the internal coherence of the plan to support shared situational awareness and thereby flexibility at the level of execution. The British model also emphasized the belief that reducing the number of assumptions decreases risk. Overall, the American ideal plan serves a controlling function, whereas the British ideal plan supports an enabling function. Interestingly, both the U.S. and UK planners viewed the other's ideal plan as riskier than their own. The implications of cultural models of plans and planning are described for establishing performance measures and designing systems to support multinational planning teams.

\section{Introduction}

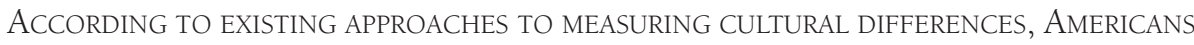
and Britons are quite similar. As an example, Hofstede's (2001) seminal studies resulted in the documentation of only very subtle differences between the populations of these two nations along the dimensions of power distance, individualism/collectivism, and so forth. Still, when U.S. and UK planners interact with each other in operational contexts, they experience the repercussions of substantive differences. The following quotation is an excerpt from an interview with an American campaign planner about his experience working with a planner from the United Kingdom:

We worked with the Brits on doing a plan for security for Pope John Paul ... and that was a real different experience - they have a completely 
different planning process ... I think it's a planning process. They do something anyway; it is far different from what the Americans do. We kind of kept looking at them like, "are you going to plan this yet?" "Oh don't worry about it, it'll come together." "Do you plan this stuff?" Where the American planning process is slightly anal retentive to most of the world, theirs is a lot more kind of, "Oh it'll come together, don't worry about it." I don't know if they did it just to drive the Americans crazy or what, but it worked. It just kind of drove us nuts.

The following quote, an excerpt from an interview with a British campaign planner, describes his experience in developing a plan for an American commander:

We did a PowerPoint presentation, a map, and we showed where there were cut offs here, here, here, and here. And [an American] three-star general came back and said, "I think two of your cut offs are a little far from each other." And we said, "Well we won't position them like that, we've just shown you dots, you know." . . . But the three-star general said, "No, prove to me, I want to see." We had to go back, put actual grid references. ... I'm not criticizing, that's the way they do it ... they have a higher level of detail. Whereas we would say to guys, "Hey, we want you to do a cut off here," which means, "Stop enemy coming out, stop people coming in." Where do you position yourself?, "Hey, when you get on the ground, you find the best spot, I can't tell you from a map, because I don't know."

An implication from these planners' experiences is that there may be culturally different ways of thinking about and approaching planning that can potentially lead to conflicts in multicultural collaborations. Their observations are consistent with work suggesting that there are significant differences in how coalition partners plan and make decisions (Sieck \& Patel, 2007). We propose that some of the cultural challenges experienced by coalition planning teams are rooted in differences in cultural knowledge relevant to the domain of planning. Before discussing such challenges further, we will first clarify the definitions and view of culture and cultural knowledge adopted here.

A scientific challenge to understanding the effect of cultural diversity on plans and planning involves generating sound definitions of the construct of culture as well as methods for analyzing and representing it. Within cognitive anthropology, culture is typically defined in terms of shared knowledge (Garro, 2000). For example, as Strauss and Quinn (1997) noted, "Cultural schemas differ not at all from other schemas learned from humanly mediated experiences, except in being shared. Schemas unique to individuals are built up from idiosyncratic experience, while those shared by individuals are built up from the various kinds of common experience" (p. 122).

More recently, this definition has been refined to account for the fact that knowledge and ideas tend to be distributed somewhat unevenly among members 
of cultural groups. This refined conception of cultural research as investigating knowledge distributed among populations is gaining acceptance among culture and cognition theorists, largely under the label of the epidemiological view (Atran, Medin, \& Ross, 2005; Sperber, 1996). The epidemiological view on culture addresses the widely shared ideas (i.e., the concepts, causal beliefs, and values) that are distributed among members of a population. Working within this theoretical framework, researchers have developed methods known as cultural network analysis (CNA), to elicit, analyze, and represent cultural knowledge in the form of networks of prevalent ideas (Sieck, Rasmussen, \& Smart, in press).

CNA represents an interdisciplinary synthesis of methods drawn from the fields of cognitive anthropology, cultural psychology, naturalistic decision making, and decision analysis (e.g., Bostrom, Fischhoff, \& Morgan, 1992; Garro, 2000; Hoffman, Crandall, \& Shadbolt, 1998; Kleinman, 1978). CNA interviews are designed to elicit the mental models from members of a particular cultural group situated within a specific domain. The consensus of individuals' mental models constitutes a cultural model for that group. CNA methods employed to investigate American and British cultural models of plans and planning represent aspects such as participants' beliefs about causal factors that influence plan quality, consequences of low- or highquality plans, and procedures and tools intended to support planning. Given this framework for understanding and modeling cultural knowledge, why should conceptions of plans and planning be expected to vary across cultures?

As the introductory quotes illustrate, people do have different ideas about what a "good" planning process looks like. The military decision-making process (MDMP), the U.S. Army's doctrinal prescription for making decisions and formalizing these into orders, represents one perspective on an ideal planning process (see U.S. Army, 2005). Research in the field of naturalistic decision making provides evidence that other conceptions of "good" planning processes exist.

For example, Schmitt and Klein (1999) demonstrated that doctrine does not provide a reliable basis for predicting what a planner's beliefs and values are with respect to an ideal planning process. Schmitt and Klein conducted a series of studies examining the informal and intuitive planning strategies skilled U.S Army and Marine Corps planning teams use. They concluded that planners rarely follow the formal, prescribed MDMP. Schmitt and Klein reported that planners, instead of generating a number of alternative courses of action as prescribed by the MDMP, often created only one course of action, which was then continually modified and improved as new information would come in. They termed this approach the recognitional planning model (RPM) because of its consistency with recognitionprimed decision-making models, in which individuals use their experience to rapidly understand situation characteristics and evolve a single decision response based on that understanding.

Formal empirical evaluations of the prescriptive value of intuitive planning models, such as the RPM relative to the MDMP, have shown that intuitive models permit an increase in planning tempo and lead to plans that are more bold and better adapted to situational demands (Blendell, Molloy, Catchpole, \& Henderson, 
2002; Pascual, Blendell, Molloy, Catchpole, \& Henderson, 2001; Thunholm, 2005). In the case of the intuitive operational planning process (IOP), as compared with the existing Canadian operational planning process, IOP may foster greater collaboration and commander involvement in planning (Bryant et al., 2007). In fact, Thunholm (2006) employed the RPM as a basis for developing a formal planning model, the planning under time pressure (PUT) model, which is currently employed as a tactical-level planning model within the Swedish Armed Forces. In contrast, research on multinational collaboration suggests that U.S. military planning processes are quite flexible compared with Japanese military planning (McHugh, Smith, \& Sieck, 2008).

There is also a diverse set of perspectives within the military community as to what a "good" planning outcome, or operational order (OPORD), looks like. Hoffman and Shattuck (2006) conducted an extensive evaluation of U.S. Army OPORDs and concluded that these orders are not consistent in how they present intent - that is, information about intent was not always in the same place and sometimes was even absent. The different models of the planning process and different values with respect to the content of planning products documented in these research efforts suggest the existence of different conceptualizations of plans and planning among professional military planners.

In addition to conceptual variations that have emerged in empirical research on planning, there are some reasons to expect differences between based on a comparison of U.S. and UK policies and doctrine. The U.S. Department of Defense established the Office of Force Transformation (OFT) in 2001 to ensure a competitive military advantage following the September 11 attacks. The OFT plan outlines a plan for changing the military force from the bottom up. This plan focuses on improving the military's capacity for joint action through the development of joint doctrine and technologies for increased information access and information sharing at all levels. This vision is captured under the rubric networkcentric operations.

The network-centric or information-centric approach in the United States has led to increased reliance on information technologies. It is possible that this trend has resulted in a command system that tends to suppress decentralized decision making. In the United States, those at the major command levels have easier and faster access to information, which may lead them to feel more confident about their decision making and thus more reluctant to disperse decision making down the command hierarchy.

The United Kingdom, in turn, has generated policies that explicitly place great emphasis on the notion of agility as part of its future defense vision (Ministry of Defence, 2003). Rather than being a network-centric approach, the UK policy focuses on human-centric attributes by regarding agility as an attribute of an individual's thinking, as demonstrated in the UK Joint High Level Operational Concept (Ministry of Defence, 2005): "essentially a human-centric attribute epitomised by the enduring ability of our people to think creatively, to be resourceful and imaginative and to adapt with versatility to the unexpected" (p. 6.2). 
Inherent in the notion of agility is the need to avoid overdependence on centralized command structures. The idea is that commanders should be able to specify strategic and operational goals without specifying how such objectives are to be accomplished. In the same publication, the UK Ministry of Defence outlined its vision for such increased decentralization of decision making: "This type of command process will be characterized by light corrections on the 'command tiller' to establish synchronization of subordinate action with higher level intent, followed by immediate restoration of freedom of action to the lowest possible levels" (Ministry of Defence, 2005, p. 2.4).

These policy descriptions suggest that British planners' shared conception of a good plan may reflect a generally more positive attitude toward decentralization, as compared with that of U.S. planners. However, the situation is not entirely clear. As mentioned in the Schmitt and Klein (1999) work described previously, experts' mental models do not always conform to doctrine and policy. Empirical research with experienced military planners is needed to determine whether there are real differences between U.S. and UK cultural models of plans and planning as well as to specify the nature of variations found.

The aim of the current research was to determine whether there are systematic cultural variations between U.S. and UK cultural models of planning. A cultural model of planning comprises a group's consensus on plan-relevant concepts as well as their beliefs about the causal relationships between concepts-that is, the antecedents and consequences of planning activities and their outcomes.

\section{Method}

\section{Participants}

The study participants were 14 experienced U.S. and UK campaign planners, 6 at Fort Leavenworth General Staff College in the United States and 8 at Cranfield Defence College in the United Kingdom. All the U.S. planners were lieutenant colonels, and the UK planners held the equivalent rank in their respective armed forces branch. All 6 American planners were in the U.S. Army; 4 of the UK planners were in the British Army, 3 were in the Royal Air Force, and 1 was from the Royal Marines. All planners had between 18 and 33 years of experience in the military (U.S. average $=19$ years, UK average $=25$ years). Four of the planners were stationed abroad at the time they were interviewed and, we felt, were more likely to provide responses that were colored by their recent experiences operating within a foreign planning environment. We also selected 10 interviews (5 American and 5 British) for analysis in which the planners had been interviewed in their country of origin.

\section{Procedure}

Each planner was interviewed individually using the same CNA interview guide. For each question, interviewees were asked to think back to one or more particularly memorable planning experiences that they had encountered in the 
course of their duties. The purpose was to ground their thinking in specific experiences and thereby ensure the validity of their responses (Crandall, Klein, \& Hoffman, 2006).

A primary and a second interviewer were present for all interviews. The primary interviewer was responsible for covering the questions in the interview guide. The second interviewer was responsible for taking notes and asking questions of clarification. Two pairs of interviewers carried out the interviews. The interviews lasted between 1 and $2 \mathrm{hr}$ depending upon the availability of the participating planners. We recorded each interview using a digital voice recorder.

\section{Materials}

A CNA interview guide was developed to elicit individual planners' mental models of plan quality. The CNA interview probes the interviewee's understanding of the antecedents of particular states, events, or actions of interest as well as the outcomes or effects these can have within particular situations or experiences. The questions probed the concepts, causal beliefs, and values relevant to the following aspects of planning: plan quality, causal factors that determine plan quality, consequences of high- and low-quality plans, functions of plans and planning, openness to making revisions in the plan (i.e., replanning), and supporting processes and tools that lead to high-quality plans. Example questions from the guide include the following:

- Can you tell us about a time when your team had developed an especially good plan?

- What are some of the characteristics that differentiate a good plan from a poor plan?

- What's the biggest mistake that a new person makes in thinking about what a plan is?

- We have talked to some good planners who say that the plan is just a platform for improvisation, rather than to be executed as written. What has your experience told you about this statement? Examples?

\section{Data Preparation/Analysis}

We used a four-step process to code and analyze the interview data. First, we transcribed the interviews and performed a protocol analysis extraction of all references in the interviews to either the causal attributes of the planning process, the planning team, or the plan itself (Ericsson $\&$ Simon, 1984). This is an example of a reference from a UK interview: "I think if you plan too much and if you have too many contingencies, everything is fine-tuned down to the last minute, when it goes wrong, as it will, it throws you because you're so tied in to this rigid structure and you're expecting to pick up the next COMM plan [communications plan] or when it doesn't happen it throws you out even more."

Second, we translated these references into one or more propositions relating the causal relationships referred to in more simple terms. For example, the propositions resulting from the foregoing reference were "including too many contingencies leads 
to a plan with a rigid structure" and "a plan with a rigid structure limits your ability to react to unexpected circumstances." This translation process generated a total of 210 propositions relating to the causal relationships among planning components as well as definitions of planning concepts across the 10 interviews.

Next, we sorted the propositions according to which aspect of planning they addressed (plan quality, causal factors that determine plan quality, etc.). The sorting aided us in the process of creating concept maps by providing high-level structure and organization for the maps.

Fourth, and finally, we used the propositions to create two concept maps, one for the U.S. and one for the UK participants. The knowledge (i.e., concepts, beliefs, values, and connections among these) elicited during a CNA interview is effectively captured and visualized in a semantic network. In a semantic network, each node represents a concept and links are used to define relations between the concepts. We created concept map representations of the mental models of planning using the CmapTools software (Cañas et al., 2004; Crandall, Klein \& Hoffman, 2006; Novak, 1998). These two concept maps served as a representation of the union of ideas within each national group.

\section{Results}

Overall, our results indicate that the U.S. and UK participants agreed on a very fundamental notion of what planning is about. They both expressed the view that planning is about identifying an end, or a goal, figuring out ways to get to that end, and the means by which you are going to get there. However, we observed interesting differences between the ways the U.S. and UK participants appeared to think about the role of the commander, the relationship between the kind of detail and plan quality, autonomy in planning and execution, risk, and what the ultimate function of a plan is. Each of these will be discussed in more detail in the following.

\section{Concept of Commander Involvement}

A prominent difference between U.S. and UK planning processes concerns the role of the commander. The United States predominantly uses a staff-led planning process, whereas the United Kingdom employs a command-led process. In the United States, it is usually the case that the commander meets with the planning staff only during a few formally scheduled briefing sessions-one at the beginning, when the commander briefs the staff on his or her intent, and one toward the end, when the staff brief the commander on the planning solutions they have generated. In this episodic involvement, the sessions become the main opportunities for the staff to obtain the commander's guidance and his or her ultimate "buyin" (see Figure 1).

This means that presentation and explanation become a vital part of the validation process: In order to get the commander's buy-in, you have to make sure that you present it well so that he or she can understand it. As one U.S. planner explained, "presenting it was a huge part of any plan we ever made. Because 


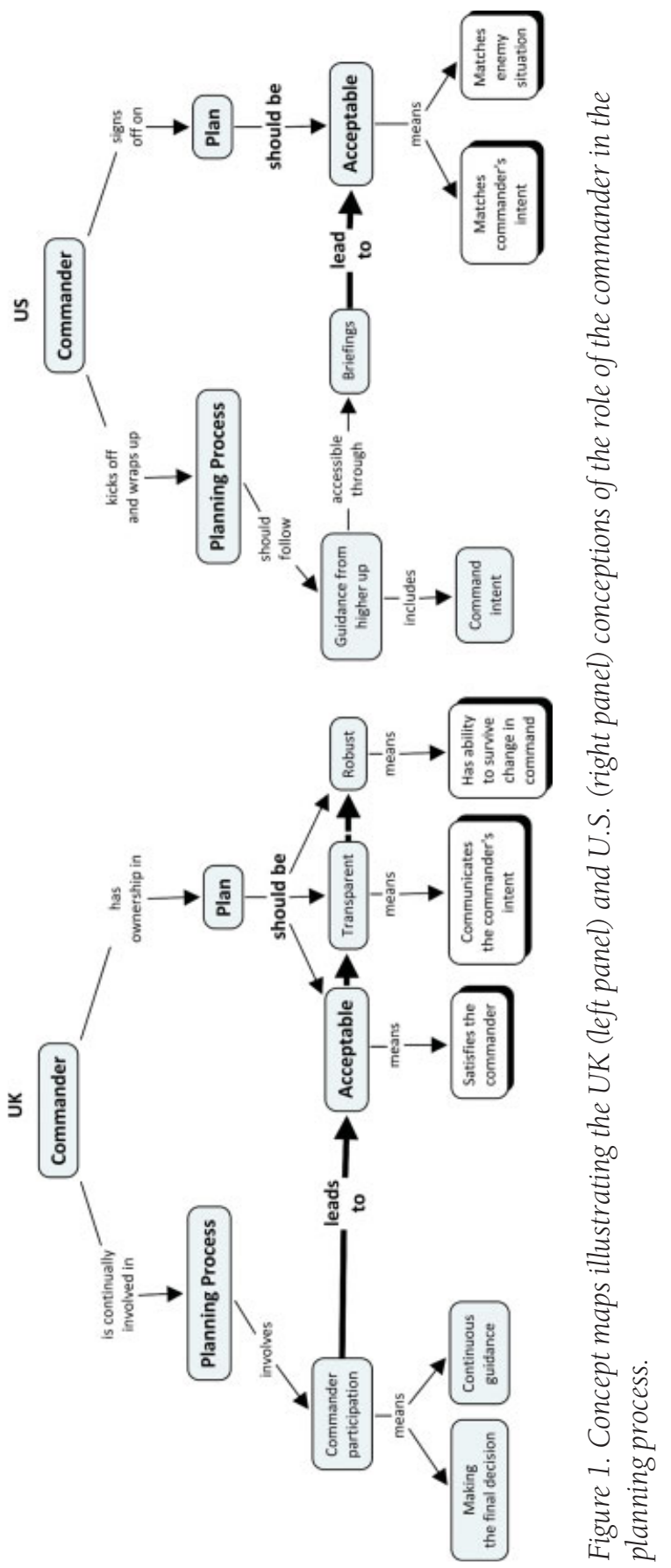


although [the commander's] a really smart guy, if he started struggling through this stuff and had to ask a lot of questions about what our thinking was, we were probably failing in explaining it properly."

In contrast, in the United Kingdom the commander plays a key role in initiating the planning process, as in the United States, but beyond that he or she is continually involved in the planning process and is far more accessible for consultation. The UK planners we talked to noted that the involvement of the commander in the planning process itself is seen as a mechanism for ensuring the commander's buy-in and satisfaction with the final outcome (see Figure 1). Because the commander is an instrumental part of creating the plan, he or she is ultimately more likely to agree with the plan.

These distinct models of commander involvement have implications for how command intent is communicated and transmitted and, ultimately, how it shapes the planning process. The episodic involvement in the United States entails that the staff have fewer, snapshot-like windows into the commander's intentions. This puts greater pressure on the formal documents that capture and communicate the commander's intent. In the United Kingdom, the staff have more continuous access to the commander, which means that they have opportunities to clarify and perhaps even negotiate the commander's intent with the commander.

The nature of the involvement of the commander in the planning process has important implications for the ways in which the planners conceptualize other important aspects of planning, such as level of detail, autonomy, risk, and plan function. Each of these aspects is discussed further in the following.

\section{Concept of Plan Detail}

Our results indicate that the U.S. and UK planners agree on the general notion that there is a relationship between the amount of detail in the plan and the overall plan quality. Both U.S. and UK planners indicated that the plan should be somewhat detailed in order to be a high-quality plan. Interestingly, however, planners from these two nations seem to have different ideas about which dimension of the plan should have a relatively high level of detail, as illustrated in Figure 2.

United States: Specifying Ways/Means to Enable Synchronization. The American model of planning specifies that a high-quality plan provides direction in terms of both goals and methods (see Figure 2). However, most of the ideas the American planners expressed had to do with providing direction on methods. They noted that a high-quality plan should specify what needs to be done as well as how it should be done-and specifying the "how" or the "ways" is particularly important. The U.S. planners expressed this idea by emphasizing the importance of working out the "how" through specifying the actions and by generating contingent methods to support meeting an objective. The implication was that if this was not done, the resulting plan would be of inferior quality.

One planner described the consequences of not providing "enough" detail: "So all of a sudden you come out of division rehearsal with a completely new plan, because you went into it with a very general plan, as opposed to a detailed plan. 


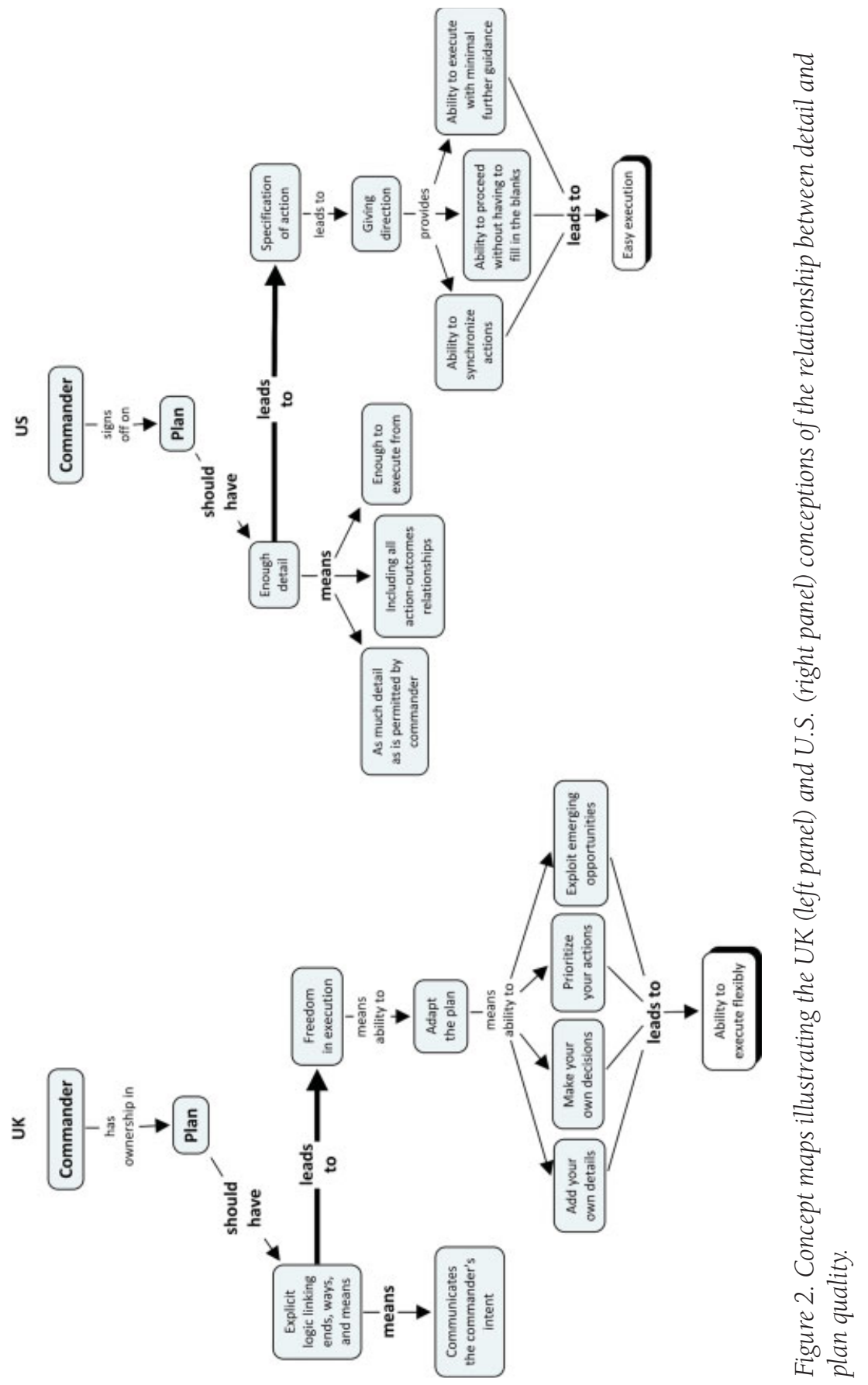


And then, all of a sudden, you know you come out of the division rehearsal and instead of being well synchronized, you come out having to write a whole new plan because subordinate commanders have all changed their minds because you didn't tell them what to do. And I've seen that happen. And as a result of a poor initial plan it was left to the subordinate commanders to kind of fill in the blanks."

From this American planner's perspective, failing to provide enough detail makes it less likely that the subordinate commanders will buy into the plan. The U.S. planners emphasized that a plan with detailed specification of actions and resources provides direction for the individuals who are inheriting the plan. Better direction, in turn, means that the plan will be easier to execute because it reduces the number of decisions that have to be made down the line. The U.S. planners therefore appeared to express the idea that a plan is a representation of decisions that have been made ahead of execution time - that is, it provides a roadmap for execution.

In fact, the U.S. planners were so emphatic about the notion that the plan should provide a roadmap that they even expressed disdain for failing to specify actions appropriately. One U.S. planner indicated that a plan lacking action specification would indicate laziness on the part of the planner. Specifically, he said, "We're going to do it in enough detail that subordinate units can execute it . . . to say that the plan is just a starting point, I couldn't disagree with you more. I think that is a cop-out for a staff that doesn't do detailed planning."

In contrast to specifying action, the UK planners focused instead on the ability of the plan to enable action.

United Kingdom: Specifying Logic to Enable Adaptation. British planners consistently emphasized that the links among the ends, the ways, and the means are more important than providing a lot of detail on the ways and means (see Figure 2). Specifying these logical linkages, or the rationale, is also more important than generating a great many contingencies (i.e., providing alternative ways and means).

One British planner described a specific planning experience that provided a particularly detailed account of the importance of preserving the logic within a plan and what it means to do so: "I was able to comprehensively, convincingly demonstrate the etymology of the plan, the genesis of the plan linking it from the policy, which I had been given, and I had no input to the policy itself, how we broke that down into a set of conditions and a strategy. How that could be developed and implemented in terms of money and time, programming. And then how that could be realized in a plan. And once l'd got that into the minds of the command group, then they were comfortable in terms of there were no holes in that deductive, rational, analytical thought process."

The UK planners indicated that the logic of the plan, when made explicit, can serve to communicate the plan's intent (i.e., the commander's intent). They further indicated that if the plan fails to demonstrate the logic, then whoever inherits the plan will be less likely to pick up the intent. If a subordinate fails to inherit the intent, he or she will be less likely to be able to adapt and execute flexibly, in a 
manner that is in line with the intent. The UK planners emphasized that having the ability to execute flexibly and still satisfy the commander's high-level objectives is paramount to success in a dynamic operating environment.

Our conclusion is that instead of seeing the plan as a record of decisions made by the planning team, the UK planners see the plan as a representation of the planning team's decision making. That is, the plan should contain the reasons behind, or the rationale for, selecting a particular strategy or course of action in order to achieve the commander's intent. The UK planners, in fact, were adamant about explicitly representing the logic or rationale for decisions made in the plan. They talked about a good plan as one that was "transparent" - that is, that anyone who picks up the plan can recognize the high-level intent. As one planner put it, "The articulation of the commander's ideas and his intent, as long as that thread is throughout the whole plan, that makes it transparent."

The UK planners further emphasized that a plan with a clear logic, or clear statements of rationale, allows the individuals who are inheriting the plan to understand the link between the commander's intent (the high-level guidance) and the ways or methods outlined in the plan. The UK planners emphasized that a plan that provides a solid platform will lead to more successful execution because it enables the individuals who are executing it to make their own decisions.

Overall, the U.S. planners indicated that a plan should specify action at some adequate level of detail. The UK planners, on the other hand, emphasized that a good plan should have sound and coherent logic. The U.S. planners, in turn, did not mention logic or rationale as an important, understandable attribute of the plan. The UK planners tended to see detailed specification of action as a constraint on flexible execution. They saw detailing of the logical relationships as a way to facilitate decision making on the part of those who are executing the plan. When the UK planners talked about specification of action, it was in the context of how detrimental overspecification could be for the flexibility of their colleagues who would be inheriting the plan.

The different ways of satisfying the commander implied by the command-and staff-led processes have differential implications for the level of detail (i.e., the number of contingencies that are included in the plan). If the planners have less insight into the commander's intent, as is the case in the United States, then they may need to add more contingencies in the hope of generating a solution that matches the commander's vision.

\section{Concept of Plan Function}

Both the U.S. and UK planners emphasized mission analysis as an important phase in the planning process. The purpose of mission analysis is to obtain an indepth understanding of the current situation, the enemy, and so forth. In that sense, for both the U.S. and the UK planners, certain aspects of the planning process are about increasing awareness of the problem that is being addressed. However, the planners did not appear to converge on the function the final plan serves for the individuals who are executing it, as illustrated in Figure 3. 


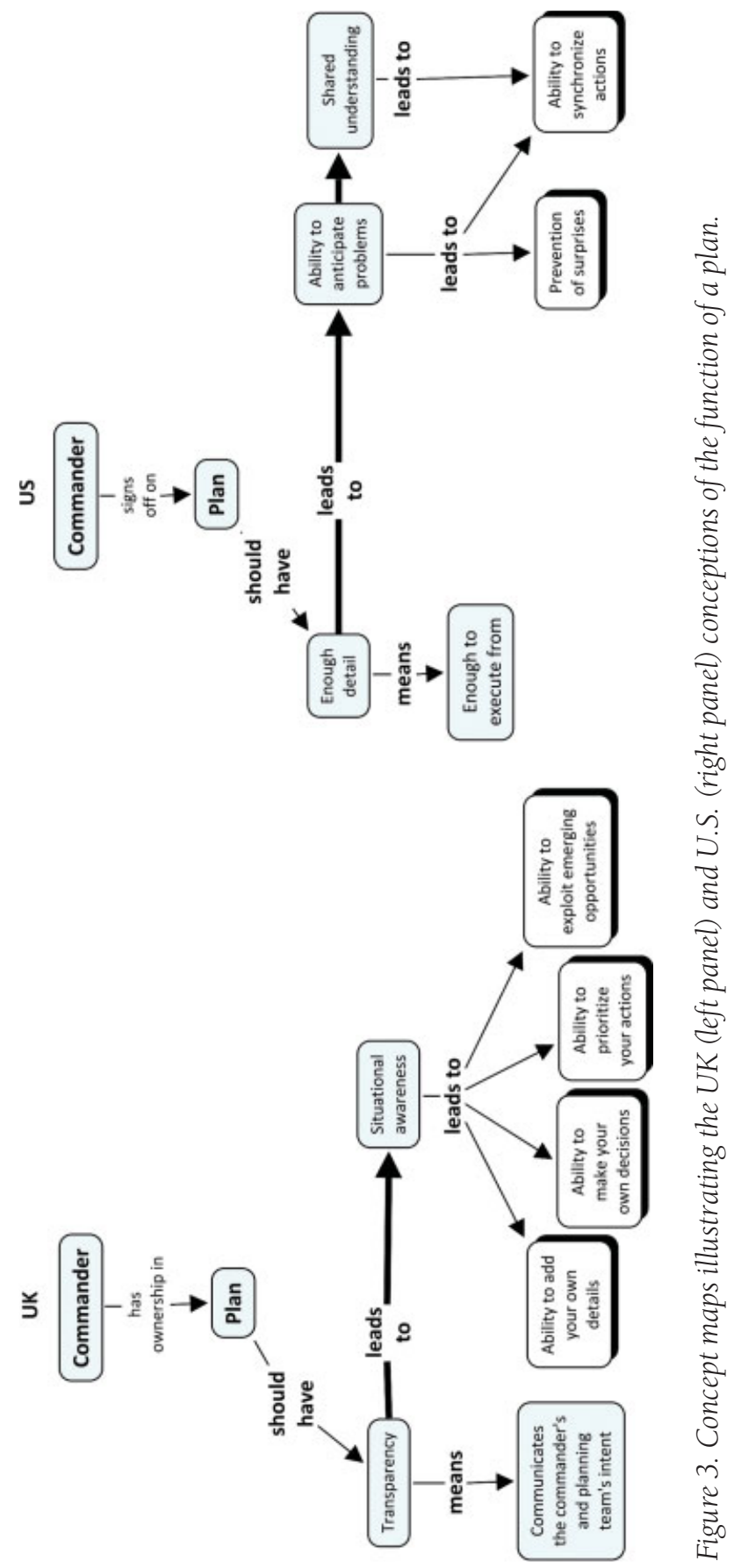


United Kingdom: Plan as Foundation for Awareness. The UK participants emphasized plans as providing shared situational awareness (see Figure 3). This is consistent with the general focus among the UK planners on enabling the individuals who are executing the plan to be able to execute it flexibly. The goal here is for the plan to provide a solid understanding of what the problem is and what the envisioned end state or goal is. Once the executors have a common understanding of what the goal is, they would be able to react to changes in the environment, adapt the plan, and still execute a coordinated effort.

United States: Plan as Foundation for Synchronization. The U.S. planners appeared to focus on the plan as a way to ensure synchronization of actions (see Figure 3), as illustrated by the following interview excerpt: "But to just say, 'Hey, we'll just write a very generic plan, giving some overall guidance, and have junior commanders figure it out.' No, because when you start to do that and then all of a sudden those enablers aren't synchronized, and you have units that are executing out of synchronization from each other. You have units that are putting out different messages; for example, you're putting out a PSYOP [psychological operations] product in this area of town, and this area of town you're putting out the complete opposite. So, I think that is a cop-out for a staff that's not doing their job."

Overall, the UK planners seemed to have the view that plans serve an enabling function, whereas the U.S. planners view plans as serving a controlling function. The goal in both cases is unity of effort. However, the two different conceptions of how to go about attaining this have very different implications for both the planning process and the final planning product.

\section{Concept of Autonomy}

Mission command in the United States as well as the United Kingdom advocates the view that staff at all levels need to have flexibility to exploit opportunities and respond to threats. The planners we interviewed all appeared to support a certain amount of autonomy. However, the UK planners in general expressed the view that autonomy is necessary at all levels of the command hierarchy. The U.S. planners talked more about autonomy at the level of planning and presented arguments against autonomy at the level of execution. The U.S. and UK cultural models of autonomy are presented in Figure 4.

United Kingdom: Freedom in Execution. As outlined in the previous section, the UK planners regarded the fundamental function of a plan as providing a foundation for flexibility in execution. It seems that UK planners place a great deal of value on freedom in execution (see Figure 4). It is possible that the UK planners simply do not need to talk about freedom in interpretation of intent, as stressed in the United States, because their commanders are more involved in the planning process.

United States: Freedom in Interpretation of Intent. The U.S. planners spoke instead of freedom in terms of interpreting the commander's intent (see Figure 4). In fact, the U.S. planners appeared to construe "freedom in execution" rather negatively. 
UK

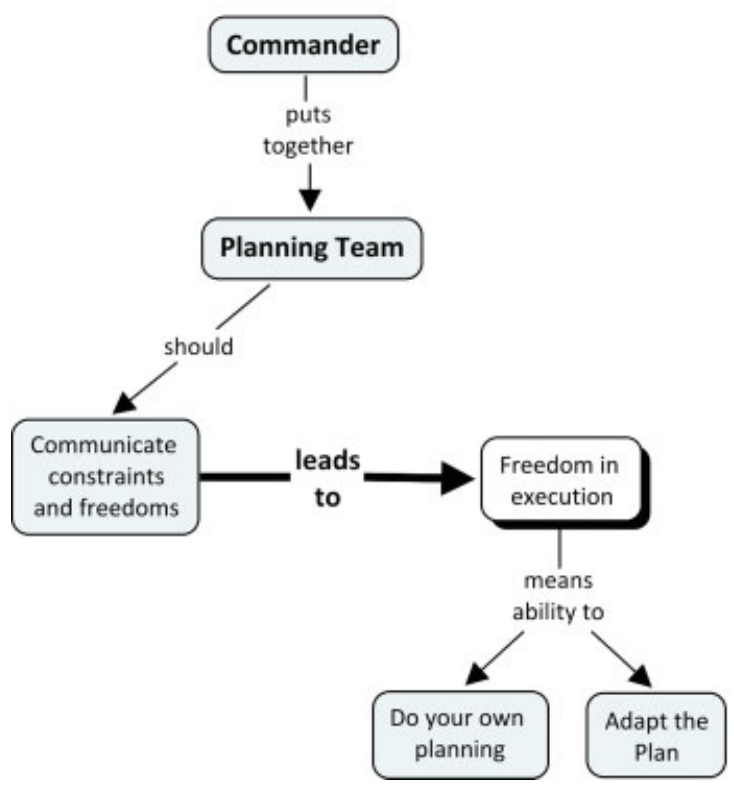

US

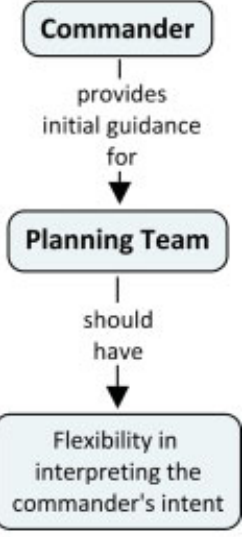

Figure 4. Concept maps illustrating the UK (left panel) and U.S. (right panel) conceptions of autonomy in planning.

They spoke of the absence of constraints in terms of requirements to make decisions, rather than as an opportunity to adapt to circumstances.

\section{Concept of Risk}

Both the UK and U.S. planners appeared to see a relationship between the number of contingencies that are included in a plan and how risky the plan is, related to the fact that each contingency brings in its own assumptions. However, the UK planners seemed to think that having more contingencies increases risk, whereas the U.S. planners thought that having more contingencies decreases risk. The models for risk are illustrated in Figure 5.

United Kingdom: Contingencies Increase Risk. The UK participants emphasized that making too many assumptions increases risk. This is related to the amount and kind of detail to include in plans. Generally, adding more detail about actions was seen as increasing the number of assumptions in the plan, which leads to increased risk (see Figure 5). One UK planner in particular talked in detail about the risks involved in making assumptions and adding contingencies to the plan. The quote appeared previously, in the Data Preparation/Analysis section, but bears repeating in the present context: "I think if you plan too much and if you have too many contingencies, everything is fine-tuned down to the last minute, when it 


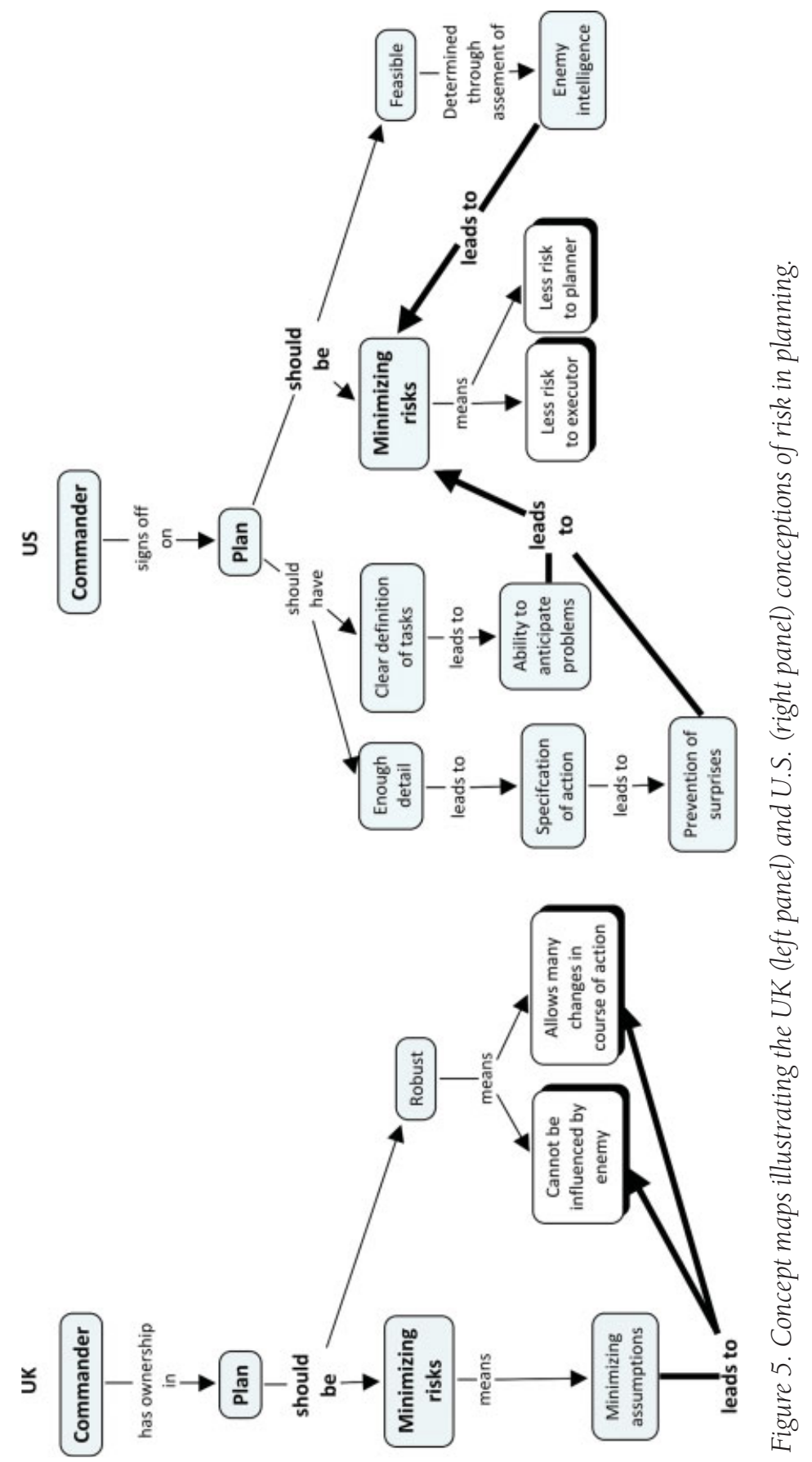


goes wrong, as it will, it throws you because you're so tied in to this rigid structure and you're expecting to pick up the next COMM plan [communications plan] or when it doesn't happen it throws you out even more."

United States: Contingencies Decrease Risk. The U.S. planners talked about risk minimization in terms of preventing surprises (see Figure 5). We heard from the UK planners that they perceive the U.S. planners as relying more on intelligence. Indeed, the U.S. planners did talk about the relationship among seeking intelligence about enemy actions, matching the plan to enemy actions, and how "risky" the plan is. The implication is that the more intelligence the planners consider, the more likely they are to add contingencies so as to reduce risk.

According to the UK model of planning, adding contingencies means making more assumptions and, therefore, increasing risk. The U.S. model, in contrast, implies that a less detailed plan is riskier. For example, one American planner said explicitly, "Risk aversion leads to a very detailed plan." The implication of this finding is that U.S. and UK planners would agree on risk reduction as a fundamental value for plans. However, differences in their causal beliefs pertaining to the antecedents of plan risk would lead each of them to judge the other's plans as unduly risky.

\section{Indicators of Plan Quality}

There is no clear universal consensus on what constitutes a high-quality plan. The U.S. and UK planners agreed that you really cannot know whether or not you have a good plan until after it has "made contact." That is, you have to implement a plan in order to know whether it is a good one. Planners from the United States and the United Kingdom, however, did not have the same ideas about what indicators to look for, during planning and as the plan is being implemented, in order to determine whether or not the plan is good. The U.S. planners tended to talk about synchronization as an indication that a plan had been implemented successfully. That is, were the specified actions carried out at the right time and in the right order? The UK planners, however, seemed to focus on adaptation instead of synchronization. In this case, did the plan enable making the right decisions at the right time and appropriately adjusting actions to evolving circumstances?

The results provided here are summarized at the level of the cultural group. There were individual planners who expressed ideas that were inconsistent with the cultural models outlined for their nation in this paper. To obtain an indicator of the level of inconsistency within each model, we divided the ideas into the five categories and performed a one-by-one comparison of each statement with every other statement within each category. From this we determined the relative number of contradictions for each model. In the U.S. data, $6.3 \%$ of the ideas had an expressed opposite. In the UK data, 3.7\% of the ideas had an expressed opposite. This means that the preponderance of ideas expressed by U.S. planners was consistent with the cultural model outlined for the United States, and the same was true for the UK planners. 


\section{Discussion}

The present study revealed several cultural differences between American and British conceptions of plans and planning. In summary, UK planners tended to emphasize the plan's logic (internal consistency and coherence), whereas U.S. planners regarded the plan as a vehicle for providing direction on methods (how an objective should be achieved). The goal of planning for UK planners appeared to be flexibility at the level of execution, and they regarded the plan as a vehicle for achieving flexibility by supporting shared situational awareness. For U.S. planners, the goal of planning appeared to be synchronization, and they regarded the plan as a platform for achieving this.

The participants' thoughts on autonomy in planning were consistent with their culturally distinct models of plan function. The UK planners regarded autonomy as important at all levels of the command hierarchy. The U.S. planners, in turn, emphasized the importance of autonomy at the planning stage. Similarly, the U.S. and UK planners' cultural models of risk were consistent with their models of plan function and autonomy. The UK planners emphasized that making too many assumptions in planning increases risk. In turn, the U.S. planners believe they reduce risk by including contingencies to prevent surprises.

In this discussion, we describe some of the historical influences that provide at least a partial account of those differences. Then, we examine the effects of the cultural differences on attitudes and preferences for tools in current use. Finally, we discuss the implications of the research for the design and development of future tools to support coalition planning.

\section{Historical Influences}

Although a complete understanding of the origins of variations in cultural knowledge is a complex sociohistorical research endeavor in its own right, we describe some differences between U.S. and UK military histories that may have led to some of the contemporary variations in mindset reported here.

British military history reveals a long-standing tradition for emphasizing adaptation. In an annex to a British Army Doctrine publication, Senior Lecturer in War Studies Dr. Brian Holden Reid (1995) noted, "if there is one word that sums up the British command style, it is improvisation" (p. 45). Reid went on, "a universal expectation prevails, almost operating like a law of nature, that a Wellington or a Montgomery, will turn up and ... turn defeat into victory" (p. 45). British commanders are therefore expected to be skilled improvisers. The basic assumption is that things will fall apart, and the commander is expected to be able to patch them up again.

This ability to "patch things up" within the British system of thought is enabled through a strong commitment to mission command. Mission command was practiced spectacularly, and even sometimes attributed to, Admiral Lord Nelson, one of the most significant British military icons of all time. Mission command is a leadership style in which command and control is exercised through consultation 
and coordination rather than hierarchical obedience. The idea is that subordinate commanders, if provided a thorough understanding of their superior commander's intent, are able to instinctively adapt to inevitable changes in the operating environment and still act in accordance with their commander's intent. As one of the British interviewees put it, "It goes back to Nelson. It's that Nelsonian touch; ... his commanders knew, instinctively, 'Okay, if this breaks down or that's happening, or we lose this, actually, it's very clear what I would need to do under those circumstances."

Interestingly, this embedded flexibility in the British system of command and control invites the interpretation that the British are ill-prepared. In fact, to quote another historical icon, Admiral Lord Chatfield, who was minister for Co-Ordination of Defence in the 1930s, "it was traditional British policy never to be ready and to be rather proud of it" (Reid, 1995, p. 44).

Col. Christopher Paparone (2001) provided an in-depth analysis of the development of what is called the military decision-making process (MDMP) in the United States. He pointed to the industrial age's focus on technology and German strategic ingenuity as having an important influence even on contemporary American military thinking: "During the industrial age, military theory began viewing armies as machines of the nation-state. Detailed algorithms of mobilization, rail schedules, and troop movements became the business of army staffs as keys to decisive victories. ... [The] Germans proved that an army that could plan detailed requirements, orchestrate capabilities rapidly and implement them precisely would win large-scale wars of national mobilization" (p. 45).

Col. Paparone (2001) argued that the current U.S. Army culture places considerable value on the analytical (procedural) aspects of the MDMP, exemplifying a third-generation warfare mentality. (Third-generation warfare employs high-speed armor and movement on the battlefield with the objective of physically destroying the enemy. Fourth-generation warfare, in contrast, has a goal of collapsing the enemy internally rather than physically destroying it, which means that targets include things such as the population's support for the war and the enemy's culture.)

Historically, the ambition toward comprehensive analysis has been a consistent pursuit within military planning and decision making as well in the development of wartime policies. Vietnam War-era Secretary of Defense Robert S. McNamara was one of the most illustrious front men in the quest for comprehensiveness. McNamara pioneered the institution of computerized, statistical methodology for conducting systems analysis, enabling the consideration of a much greater set of contextual factors than had previously been possible (Grattan, 2006). It was the aim that every decision could be considered in as broad a context as necessary. Notably, McNamara's computerized methodology forecasted that the Vietnam War would end within a reasonable period of time. The fervor with which computational models that can assist in the prediction of effects across the spectrum of operations are pursued, and the fervor with which technological solutions to complex problems are pursued in general, remain prominent within the United States. 
These various historical precedents constitute initial explanations of the distinct U.S. and UK cultural models of plans and planning, and we recognize that future research is needed to fully explicate the myriad causal factors involved. Next, we examine the effects of the distinct models on preferences for plan representation formats and tools.

\section{Implications of Cultural Differences for Tool Usage}

One potential implication of the differences between the U.S. and UK conceptualizations of planning is a difference in the general attitudes toward tools and their role in the planning process, as well as tendencies to use different tools to develop and represent plans. For example, Americans use Microsoft PowerPoint to capture and brief plans, whereas Britons tend to use Microsoft Word documents. This makes for an important difference between the two nations' work processes. In the interviews, the U.S. planners expressed strong opposition to using Word, whereas the UK planners expressed the opposite sentiment. We suggest that a potential reason the UK planners show strong preferences for using Word is that this format is better for capturing the relationships between stages of thinking in the planning process. To support this notion, they refer to U.S. thinking as "PowerPoint deep," meaning that the method that Americans use to physically represent and brief their plans constrains them to a "shallow" way of thinking about the plan.

One UK planner described a specific experience he had while working with Americans when he was obliged to brief the American commander using PowerPoint. He indicated a preference for capturing the logic, or rationale, using Word: "I could give them the PowerPoint deep bit, which was woven together, but if they wanted to do root canal surgery I always had the team in the wings who could come out with the detail that underpinned my PowerPoint brief. But what we never had the time to do in that 3 weeks was turn that PowerPoint into a Word document, a seamless policy, strategy, program, plan."

It is possible that because Word allows you to write in coherent expository form, you can capture the relationships between the intent and the strategy, for example, in a way that the bullet-point format in PowerPoint does not. Basic cognitive research has shown that exposition does, in fact, affect reasoning and decision processes, consistent with this claim (Sieck \& Yates, 1997).

\section{Implications of Cultural Differences for Technology Design}

The current study has a number of implications for the development of future technologies to support coalition planning. First and foremost, the results affirm the supposition that technology development initiatives should be sensitive to the concepts, causal beliefs, and values of different cultural groups. In terms of technology design, we propose that designers will be able to assess commonalities and differences between two or more cultures based on CNA's formal representations of culturally shared knowledge. Comparing the cultural models can enable the 
designer to build affordances into the system that allow it to accommodate and support diverse cultural models of the planning task.

Our results indicate that U.S. and UK planners are essentially solving different problems: For example, the Americans are developing detailed blueprints for action, whereas the Britons are creating plan representations that can support the effective communication of command intent. Our results also indicated that although the U.S. and UK planners agreed that one really cannot know whether or not one has a high-quality plan until after it has "made contact," prior to execution they do look at very different aspects of the plan when they evaluate quality. As we outlined, U.S. planners pay attention to the level of detail that the plan provides on the methods that should be used for enacting the plan. UK planners, in turn, focus on whether or not the plan explicitly outlines the logic between methods and goals. Based on this, the functionality of coalition-planning tools should be differentially suited to cultural groups and their particular way of parsing the problem space. This means that differences in cultural models could translate into design considerations with respect to

- the opportunities for user interaction afforded by the tool;

- the kind of information made available to users at different stages of the planning process;

- the support for creating, defining, and editing different types of information; and

- the ease with which users can assess whether or not a given plan meets certain cultural criteria for quality.

We suggest that in order for a planning tool to support both cultural models of the relationship between the planning process and plan outcomes, it should accommodate a planning process in which much attention is given to detail on methods and a process whereby the logic between methods and goals is elaborated in detail. Further, the tool should make it easy to assess whether or not a given plan meets these two cultural criteria for quality. We propose that when the system supports these diverse cultural models of the task, it will allow both U.S. and UK users to share knowledge and coordinate their cognitive work. This could perhaps be achieved through improvements to the operational orders (OPORD) component of the planning process.

The OPORD is essentially the document through which echelons communicate intent up and down the command hierarchy. Empirical work by Hoffman and Shattuck (2006) has suggested that the OPORD process could be enhanced by allowing the expression of OPORD information in meaningful diagrams, such as concept maps. Diagrams support cognition in a number of important ways-among others, by shifting some of the information-processing burden onto the visual perception system. Diagrams such as concept maps are flexible with respect to the level of detail that can be included, and they make it easier to see the logical linkages between planning elements, including the commander's intent, and thus appear to satisfy requirements from both planning communities sampled in the current study. 
Another avenue through which technology has the opportunity to support multinational planning team performance is through intelligent assistive functions that support the development of plans that are acceptable along multiple evaluative dimensions. In order to support machine-based, culture-sensitive evaluations of plan quality, a common representational scheme is needed for capturing the contents of the cultural models themselves-for example, to be able to define and represent what it means for something for to be a good or acceptable plan from a specific cultural viewpoint.

One way of explicitly representing the content of cultural models is to capitalize on the technological outcomes of the semantic Web initiative (Berners-Lee, Hendler, \& Lassila, 2001). Ontology languages such as the Ontology Web Language (OWL) could be useful here because they provide a means to create conditions for category membership based on the properties of plans and their component parts (Antoniou \& van Harmelen, 2004; McGuiness \& van Harmelen, 2004). One direction for future research is to explore the potential of OWL to represent cultural knowledge, perhaps through the use of the CmapTools extension called CmapTools Ontology Editor (COE), which translates resource description framework (RDF) and OWL to and from concept maps (Eskridge, Hayes, Hoffman, \& Warren, 2006).

\section{Conclusion}

The changing nature of contemporary military engagements has led those in Western militaries to rethink important aspects of how they conduct operations. Some have argued that the problems inherent in modern conflicts, such as countering insurgencies or combating terrorism, require a new conception of coalition planning. One line of research suggests that solving the kinds of complex, "wicked" challenges that face the global community today requires a diversity of perspectives and expertise, which can be applied only through effective collaboration between culturally dissimilar coalition partners and local delegates (Pierce \& Dixon, 2006). Including all of the relevant parties "at the table" appears to be insufficient, given that even very culturally similar partners such as the United States and the United Kingdom have different views of how to proceed in making decisions and plans. In addition, planning processes and systems must be developed that can be flexibly adapted to operate well with the culturally distinct decisionmaking customs of all of the coalition partners involved.

\section{Acknowledgments}

This research was sponsored by the U.S. Army Research Laboratory and the UK Ministry of Defence and was accomplished under Agreement Number W911NF-06-3-0001. The views and conclusions contained in this document are those of the authors and should not be interpreted as representing the official policies, either expressed or implied, of the U.S. Army Research Laboratory, the U.S. government, the UK Ministry of Defence, or the UK government. The U.S. and 
UK governments are authorized to reproduce and distribute reprints for government purposes notwithstanding any copyright notation hereon.

The authors gratefully acknowledge the valuable comments and contributions of Jitu Patel, Ian Whitworth, David Roberts, Rob Hutton, Elizabeth Veinott, and Jennifer Smith.

\section{References}

Antoniou, G., \& van Harmelen, F. (2004). Web Ontology Language: OWL. In S. Steffen \& R. Studer (Eds.), Handbook on ontologies (pp. 67-91). Berlin: Springer Verlag.

Atran, S., Medin, D. L., \& Ross, N. O. (2005). The cultural mind: Environmental decision making and cultural modeling within and across populations. Psychological Review, 112, 744-776.

Berners-Lee, T., Hendler, J., \& Lassila, O. (2001). The semantic web. Scientific American, 284(4), 34-43.

Blendell, C., Molloy, J. J., Catchpole, L. J. \& Henderson, S. M. (2002). A second investigation of alternative command planning processes (Defence Evaluation and Research Agency paper). Manuscript in preparation.

Bostrom, A., Fischhoff, B., \& Morgan, M. G. (1992). Characterizing mental models of hazardous processes: A methodology and an application to radon. Journal of Social Issues, 48, 85-100.

Bryant, D. J., Bruyn Martin, L., Bandali, F, Rehak, L., Vokac, R., \& Lamoureux, T. (2007). Development and evaluation of an intuitive operational planning process. Journal of Cognitive Engineering and Decision Making, 1, 434-460.

Cañas, A. J., Hill, G., Carff, R., Suri, N., Lott, J., Eskridge, T., et al. (2004). CmapTools: A knowledge modeling and sharing environment. In A. J. Cañas, J. D. Novak, \& F. M. González (Eds.), Concept maps: Theory, methodology, technology: Proceedings of the 1st International Conference on Concept Mapping (pp. 125-133). Pamplona, Spain: Universidad Pública de Navarra. Available from http://cmc.ihmc.us/CMC2004Programa.html.

Crandall, B., Klein, G., \& Hoffman, R. (2006). Working minds: A practitioner's guide to cognitive task analysis. Cambridge, MA: MIT Press.

Ericsson, K. A., \& Simon, H. A. (1984). Protocol analysis: Verbal reports as data. Cambridge, MA: MIT Press.

Eskridge, T., Hayes, P., Hoffman, R., \& Warren, M. (2006). Formalizing the informal: A confluence of concept mapping and the semantic web. In A. J. Cañas \& J. D. Novak (Eds.), Concept maps: Theory, methodology, technology: Proceedings of the Second International Conference on Concept Mapping (pp. 247-254). San Jose, Costa Rica: Universidad de Costa Rica.

Garro, L. C. (2000). Remembering what one knows and the construction of the past: A comparison of cultural consensus theory and cultural schema theory. Ethos, 28, 275-319.

Grattan, R. (2006). Robert McNamara's "11 lessons" in the context of theories of strategic management. Journal of Management History, 12, 425-438.

Hoffman R., Crandall, B., \& Shadbolt, N. (1998). A case study in cognitive task analysis methodology: The critical decision method for the elicitation of expert knowledge. Human Factors, 40, 254-276.

Hoffman, R., \& Shattuck, L. (2006). Should we rethink how we do OPORDS? Military Review, 86(2), 100-107.

Hofstede, G. (2001). Culture's consequences (2nd ed.). Thousand Oaks, CA: Sage.

Kleinman, A. (1978). Concepts and a model for the comparison of medical systems as cultural systems. Social Science and Medicine, 12, 85-93. 
McGuiness, D. L., \& van Harmelen, F. (2004). OWL Web Ontology Language overview. Retrieved August 8, 2009, from the World Wide Web Consortium: http://www.w3.org/TR/owl-features/

McHugh, A. P., Smith, J., \& Sieck, W. R. (2008). Cultural variations in mental models of collaborative decision making. In J. M. C. Schraagen, L. Militello, T. Ormerod, \& R. Lipshitz (Eds), Macrocognition and naturalistic decision making (pp. 141-158). Aldershot, UK: Ashgate.

Ministry of Defence. (2003). Delivering security in a changing world: Defence white paper (Rep. No. Cm 6041-I). London: Stationery Office.

Ministry of Defence. (2005). The UK joint high level operational concept (Rep. No. Joint HLOC). London: Author, Joint Doctrine and Concepts Centre.

Novak, J. D. (1998). Learning, creating, and using knowledge. Mahwah, NJ: Erlbaum.

Paparone, C. R. (2001). U.S. Army decision-making: Past, present and future. Military Review, 81(4), 45-53.

Pascual, R. G., Blendell, C., Molloy, J. J., Catchpole, L. J., \& Henderson, S. M. (2001). An investigation of alternative command planning processes (Defence Evaluation and Research Agency paper prepared for the UK Ministry of Defence). Manuscript in preparation.

Pierce, L., \& Dixon, M. (2006, May). Improving multicultural teamwork to combat terrorism. In Tactical decision-making and situational awareness for defense against terrorism. North Atlantic Treaty Organization Systems Concepts and Integration (SCI) Panel Workshop SCI174, Turin, Italy.

Reid, B. H. (1995). The British style of command. Annex to Army Doctrine Publication, Volume 2, Command (Army Code No. 71564). London: Ministry of Defence.

Schmitt, J., \& Klein, G. (1999). A recognitional planning model. In Proceedings of the Command and Control Research and Technology Symposium 1999. Newport, RI: U.S. Naval War College.

Sieck, W. R., \& Patel, J. (2007). Cultural issues in coalition planning. In J. Lawton, J. Patel, \& A. Tate (Eds.), Proceedings of the Fourth International Conference on Knowledge Systems for Coalition Operations (KSCO-2007, pp. 88-92). Waltham, MA: IEEE Press.

Sieck, W. R., Rasmussen, L. J., \& Smart, P. (in press). Cultural network analysis. In D. Verma (Ed.), Network science for military coalition operations: Information extraction and interactions. Hershey, PA: IGI Global Publications.

Sieck, W., \& Yates, J. F. (1997). Exposition effects on decision making: Choice and confidence in choice. Organizational Behavior and Human Decision Processes, 70, 207-219.

Sperber, D. (1996). Explaining culture: A naturalistic approach. Malden, MA: Blackwell.

Strauss, C., \& Quinn, N. (1997). A cognitive theory of cultural meaning. Cambridge, UK: Cambridge University Press.

Thunholm, P. (2006). A new model for tactical mission planning for the Swedish armed forces. In Proceedings of the 2006 Command and Control Research and Technology Symposium: The state of the art and the state of the practice (CD-ROM). Washington, DC: Commmand and Control Research Program.

Thunholm, P. (2005). Planning under time pressure: An attempt toward a prescriptive model of military tactical decision making. In H. Montgomery, R. Lipshitz, \& B. Brehmer (Eds.), How professionals make decisions (pp. 43-56). Mahwah, NJ: Erlbaum.

U.S. Army. (2005). FM 5-0: Army planning and orders production. Washington, DC: U.S. Government Printing Office.

Louise J. Rasmussen is a senior scientist in the Culture \& Cognition Group at Applied Research Associates. Her research focuses on cultural cognition, particularly on how culturally determined understandings of a task domain can influence behaviors, performance, and expectations in work situations. Rasmussen currently leads several research projects in intercultural 
interaction and collaborative decision making. She received a $\mathrm{PhD}$ in psychology with an emphasis on discourse analysis and human factors from Wright State University in 2007.

Winston R. Sieck is a principal scientist at Applied Research Associates, where he leads the Culture \& Cognition Group. He conducts research on culture and decision making and develops applications for cultural analysis, intercultural understanding and influence, and multicultural collaboration. Sieck received a PhD in psychology with emphasis on cognition, culture, and decision making from the University of Michigan in 2000.

Paul Smart is a senior research fellow at the School of Electronics and Computer Science, University of Southampton. His research interests include the development and exploitation of semantic technologies, the design of user interfaces to support knowledge use and sharing, and the impact of network technologies on human intelligence. Smart currently works as part of the Web Science Research Institute, which is a multidisciplinary research body seeking to undertake fundamental research into the World Wide Web. 